\title{
Ein Unterrichtsansatz zu Kleists Erzählung \\ Das Bettelweib von Locarno Entwurf einer Visualisierung für den Lese- und Literaturunterricht
}

\section{Petr Pytlík}

This paper presents a lesson design as a supplement to the traditional reading and literature lessons. The introduction briefly describes the theoretical foundations of the use of visualizations to promote learning, especially the approaches of the Dual Coding Theory. It describes the emergence of mental images during textual perception and their possible externalization. The main part of this paper introduces a concrete design for reading lessons using the example of the short story Das Bettelweib von Locarno by Heinrich von Kleist. In this paper it is assumed that the externalization of the mental imagery "in the minds" of the students is an effective complement to successful literature classes.

dual coding theory, visualization in reading and literature lessons, Heinrich von Kleist, externalization of mental imagery

Der vorliegende Beitrag stellt einen Unterrichtsentwurf als Ergänzung zum traditionellen schriftorientierten Lese- und Literaturunterricht vor. In der Einleitung werden kurz die theoretischen Grundlagen der Verwendung von Visualisierungen zur Förderung des Lernens, besonders die Ansätze der Dual-Coding-Theorie, vorgestellt. Anschließend wird der Prozess der Entstehung von mentalen Bildern bei der Textwahrnehmung und deren Externalisierung erörtert, um im letzten Teil zu einem konkreten Entwurf für den Leseunterricht am Beispiel der Erzählung Das Bettelweib von Locarno von Heinrich von Kleist zu kommen. In diesem Beitrag wird angenommen, dass die Externalisierung von inneren Bildern „in den Köpfen“ der Lernenden eine effektive Ergänzung zum erfolgreichen Literaturunterricht darstellt.

dual coding theory, Visualisierungen im Lese- und Literaturunterricht, Heinrich von Kleist, Externalisierung von mentalen Bildern

\section{Zur Einführung}

Grafiken und Bilder haben schon seit Langem Einzug in den Literatur- und Literaturwissenschaftsunterricht gehalten, besonders in den Erläuterungen der oft schwer verständlichen Paradigmen der Erzähltheorie, aber auch beim Erlernen der eigentlichen Textanalyse. Gut gewählte Bilder, Comics und Filme haben Einfluss auf die Rezeption literarischer Texte, machen Präsentationen attraktiver und fördern das Gedächtnis. Daher ist es kein Wunder, dass in den letzten Jahrzehnten Fragen der medientheoretischen und semiotischen Herangehensweise an den Literaturunterricht immer wieder in den Fokus der Kulturwissenschaften rücken (Lecke 1999; Binczek, Dembeck und Schäfer 2013; Schneider und Schöch 2014). So finden sich Studien zur Visualität von Literatur, zur optischen Poesie oder zu 
Bild-Text-Verhältnissen in literarischen Studien. Diese Publikationen sind allerdings immer noch Ausnahmen, denn im Mittelpunkt des Leseunterrichts steht nach wie vor der traditionelle Umgang mit schriftlichen Texten - das Textverständnis wird als ein Entwicklungsinstrument des abstrakten Denkens verstanden. Doch angesichts der allgemein schwachen Lesekompetenzen von SchülerInnen (und nicht nur von diesen) sowohl in Deutschland als auch in Tschechien und anderen Ländern scheint es sinnvoll zu sein, nach neuen (ergänzenden) Strategien zu suchen, die diese Kompetenzen effektiver entwickeln könnten.

Die Verwendung von neuen Strategien im Leseunterricht hängt nicht ausschließlich mit dem Stand der theoretisch-methodologischen Unterstützung der Lehrenden zusammen. Wie Tatjana Jesch und Michael Staiger in ihrem Aufsatz Bilder und Visualisierungen in der Lese- und Literaturdidaktik, der auch als eine umfassende Einführung in die Problematik benutzt werden kann, zeigen, hängt das Interesse an der Neugestaltung des Unterrichts auch von externen Faktoren ab, wie dies in Deutschland die Ergebnisse von PISA 2000 zu der sehr geringen Kompetenz deutscher Schülerinnen und Schüler im Umgang mit literarischen Texten waren (Jesch und Staiger 2016: 64). Wie bekannt führte diese Studie in Deutschland zu einer Diskussion über den bisherigen Lese- und Literaturunterricht an deutschen Schulen und zu einer intensiven Suche nach neuen Zugängen zu geschriebenen Texten. Auch an tschechischen Schulen wären solche Ansätze zum Umdenken im Leseunterricht relevant, wie u. a. auch die unterdurchschnittlichen Ergebnisse der tschechischen Schülerinnen und Schüler bei PISA 2015 zeigen. ${ }^{1}$ Der hier vorliegende Text ${ }^{2}$ ist ein Entwurf zu einer solchen möglichen Ergänzung zum traditionellen Literaturund Leseunterricht und basiert auf der Hypothese, dass eine häufigere Verwendung von Visualisierungen als eine der erlernten Lesestrategien die Lesekompetenz von tschechischen SchülerInnen inner- und außerhalb des Fremdsprachenunterrichts unterstützten könnte.

Im ersten Teil sollen die theoretischen Grundlagen der Verwendung von Visualisierungen im Unterricht, besonders die Hypothesen, Experimente und Ergebnisse der Dual Coding Theory, vorgestellt werden, die anschließend als Ausgangspunkt für einen konkreten Visualisierungsentwurf für den Literaturund Leseunterricht dienen. Außerdem wird ein kleiner Exkurs in die Problematik der Verwendung von Visualisierungen im Lese- und Literaturunterricht in englischsprachigen Ländern und in Deutschland unternommen. Im Anschluss an die Darstellung der theoretischen Grundlagen wird der konkrete Prozess der

1 Der Bericht über Ergebnisse der tschechischen SchülerInnen ist unter folgendem Link einzusehen: https://www.csicr.cz/Csicr/media/Prilohy/PDF_el._publikace/Mezin $\%$ c3\%a1rodn $\%$ c3\%ad $\% 20$ $\%$ c5\%a1et $\%$ c5\% $\% 9$ en $\%$ c3\%ad/NZ_PISA_2015.pdf.

2 Der Text ist im Rahmen des Programms zur Förderung der Entwicklung der Masaryk-Universität Brno (FRMU), Projekt-Nr. 1125/2018, Projekt-Titel: Metodický on-line portál pro učitele němčiny se zaměrením na kulturni kompetence ve výuce entstanden. 
Externalisierung von internen (mentalen) Bildern beschrieben und gezeigt, welche Vorteile die Anwendung der entsprechenden Unterrichtsverfahren für die literarische Textanalyse hat. Im Hauptteil des Textes wird dann ein konkreter Entwurf für eine Visualisierung im Lese- und Literaturunterricht vorgestellt. Der hier entworfene Ansatz, der im Folgenden detailliert beschrieben wird, setzt bei den Lehrkräften die Bereitschaft zu spontaner Anpassung ihres Verhaltens an die jeweilige Situation in der Gruppe voraus, indem er Nachdruck auf die Dynamik der jeweiligen Lernergruppe auf der Achse zwischen einem ganzheitlichen Unterricht, einem „holistic approach“, und einem auf konkrete Fähigkeiten orientierten Unterricht, einem „skills approach“, legt. ${ }^{1}$

\section{Visualisierungen ${ }^{2}$ in der didaktischen Theorie}

Visualisierungen im (Literatur)Unterricht wird in der deutsch-, englisch- und tschechischsprachigen Didaktik unterschiedlicher Wert beigemessen. Grundsätzlich kann behauptet werden, dass die englischsprachige Didaktik-Theorie eine führende Stellung bei der Erforschung der kognitiven Prozesse und der Rolle der Visualisierungen beim Lernen einnimmt. Die von Allan Paivio im Jahre 1971 konzipierte und von Mark Sadoski und Richard Mayer weiterentwickelte Dual Coding Theory (DCT) erfasst die Rolle der verbalen und visuellen Vermittlung von Informationen beim Lernen und bei deren Speicherung. In Tschechien sind die Erkenntnisse der DCT nicht besonders verbreitet, im Internet findet man lediglich eine Präsentation von Jan Hedbávný (Hedbávný 2008), die an der Pädagogischen Fakultät der Karlsuniversität Praha entstanden ist.

Grundsätzlich liegt der DCT die Annahme zugrunde, dass visuelle und verbale Informationen in zwei unterschiedlichen Hirnarealen verarbeitet werden. Deshalb führte der Begründer der Theorie, Allan Paivio, eine Reihe von Experimenten durch, in denen es vor allem um die Erprobung der dualen Kodierung in Wort und Bild und deren Einfluss auf die Gedächtnisleistung des Menschen ging und mittels derer er seine Hypothesen von der dualen Kodierung von Informationen experimentell nachweisen konnte (Paivio 1971). Auch neueste neurowissenschaftlich orientierte Studien zur Verwendung von Visualisierungen im Leseunterricht liegen vor, die mit der Überprüfung der DCT verbunden sind (Paivio 2008). Aus den Erkenntnissen der DCT zur Visualisierung von Texten wird ebenfalls die verstehensfördernde

1 Diese zwei Begriffe stammen von Allan Paivio, dem Begründer der Dual Coding Theory, und werden weiter unten im Text detailliert erklärt.

2 Der Begriff der Visualisierungen, so wie er in diesem Beitrag verstanden wird, verweist auf eine Übertragung des abstrakten Inhalts in eine ikonische Veranschaulichung, die intern oder extern erfolgt (Jesch und Staiger 2016). Diese Verortung des Begriffes der internen und externen Visualisierung ist für den vorliegenden Beitrag der Ausgangspunkt für eigene Überlegungen und zu einem textanalytischen Entwurf. 
Wirkung der Rezeption vorgegebener externer Visualisierungen bei der Lektüre theoretisch abgeleitet und empirisch nachgewiesen (Jesch und Staiger 2016: 67-68).

Die DCT-Forscher studieren primär das begleitende Visualisieren von Texten und dessen Einfluss auf die Interpretationsfähigkeit und das Gedächtnisvermögen von Rezipienten. Die Grundannahmen der DCT hat Paivio aber auch zu anderen Zwecken bei der Verwendung verschiedener Unterrichtsstrategien angewendet. Paivio befasste sich u. a. mit der Informationsverarbeitung im nonverbalen System, d. h. mit der Bildung mentaler Visualisierungen, mit der „(mental) imagery“. Aus den Experimenten von Paivio ergibt sich dieSchlussfolgerung, dass Visualisierungsprozesse durch die Verarbeitung von konkreter Sprache aktiviert werden, nicht hingegen durch die Verarbeitung von abstrakter Sprache. Wenn die Voraussetzungen für eine sowohl verbale als auch nonverbale, d. h. mit internen Visualisierungen verbundene Übertragung und Verarbeitung der verbalen Information erfüllt sind, führt dies zu einem besseren Verständnis der überlieferten Information und einer höheren Gedächtnisleistung, da elaboriertere mentale Repräsentationen gebildet werden, die einen besseren Zugang zur gespeicherten Information ermöglichen (vgl. Lenzner 2009: 83). Im Anschluss an Paivios Experimente kommt Lenzner zu der Schlussfolgerung, dass die doppelte Kodierung des sprachlichen und bildlichen Inputs das Textverständnis fördert, und zwar dadurch, dass die Textverarbeitung zugleich auf einer verbalen und einer nonverbalen imaginativen Ebene stattfindet. Aufgrund des nonverbalen Codes werden dabei die nichtsprachlichen Stimuli verarbeitet, während der verbale Code für die sprachlichen Informationen zuständig ist. „Bilder können sozusagen als eine Art Gedächtnisanker wirken, der auch den Abruf der zusammen mit dem Bild gelernten Textinformation erleichtert" (Lenzner 2009: 83). Außerdem beeinflusst das Zusammenspiel beider Systeme nicht nur die Kognition, sondern auch die Motivation, Inspiration und Emotion beim Lesen und Verstehen von Texten (vgl. Sadoski 2003: 80). ${ }^{1}$

Marc Sadoski unterscheidet zwei verschiedene Herangehensweisen an die Verwendung von Visualisierungen im Unterricht. Demnach erfolgt das Visualisieren nicht nur unter methodischer Anleitung, sondern auch spontan. Bei einem Leseunterricht, der besonders spontane Visualisierungen fördert, konnte Mark Sadoski nachweisen, dass die SchülerInnen nach der Lektüre illustrierter Texte ihre eigenen internen Visualisierungen nachträglich nicht mehr von den vorgegebenen externen Visualisierungen unterscheiden konnten und dass nichtillustrierte Texte

1 Die Verwendung von externen Bildern bei der Lektüre und die Möglichkeit, auf die begleitende Rezeption von vorhandenen Bildern zur Unterstützung des Textverständnisses zurückzugreifen, werden im vorliegenden Artikel nicht weiter behandelt. Dem Autor geht es vielmehr darum, den Verarbeitungsprozess in umgekehrter Richtung zu verfolgen, denn statt fertige, im Voraus vorbereitete Bilder als Begleitmaterial im Leseunterricht zu verwenden, werden in der hier beschriebenen Konzeption die Bilder erst im Unterricht durch die Lesenden aufgrund ihrer mentalen Bilder geschaffen. 
im Vergleich zu illustrierten Texten sogar doppelt so viele bildliche Vorstellungen evozierten (Sadoski 2001).

Entsprechend hat Sadoski die Rolle des Lehrenden im Zusammenhang mit der Verwendung von Visualisierungen im Leseunterricht in ihrer Doppeldeutigkeit und in ihrer gegenseitigen Beeinflussung als eine Brücke zwischen zwei Herangehensweisen bestimmt: dem Unterrichtsansatz, der auf Fähigkeiten des Dekodierens, des Verstehens und der Reaktion abzielt (skills approach), und dem ganzheitlichen Ansatz (holistic approach). Die Rolle der Lehrperson wird auch bei dem im vorliegenden Beitrag beschriebenen Entwurf möglichst unterdrückt. Es sollte zur gegenseitigen Konfrontation der internen Visualisierungen der einzelnen Lernenden kommen, was zur Diskussion, im Idealfall zum besseren allgemeinen Textverständnis beitragen kann.

Das einflussreiche und durch viele empirische Studien bestätigte Modell der DCT wird besonders in der letzten Zeit aus verschiedenen Positionen heraus kritisiert. Kernpunkt der Kritik ist die Behauptung, der DCT liege eine verengte Auffassung von Rezeptionsprozessen zugrunde, da die Theorie eine ziemlich deutliche Trennung zwischen den genannten Codierungen voraussetzt. Eine solche starke Trennung wird (nicht nur) von der neueren neuropsychologischen Forschung in Frage gestellt (Genc 2011). Es zeigt sich nämlich, dass die zwei Hirnareale, die für die nonverbale bzw. verbale Wahrnehmung verantwortlich sind, nicht getrennt arbeiten, sondern miteinander mittels des sog. Corpus callosum kommunizieren, und dass sich beispielsweise an der Rezeption und Deutung von visuellen Informationen beide Hirnhälften aktiv beteiligen.

DieDCTkönnteaufgrundihrerverengtenAuffassungvom Wahrnehmungsprozess auch vom Standpunkt der literaturwissenschaftlichen Rezeptionsästhetik kritisiert werden, die besonderen Nachdruck auf den ganzen Prozess der Textrezeption legt. Die kritisierte Verengung hängt mit dem starken Fokus der DCT auf die Bild-Text-Wahrnehmung zusammen, wobei weitere, eventuell ebenfalls relevante Wahrnehmungsbedingungen und Faktoren weniger berücksichtigt werden. Während also die DCT die visuelle Wahrnehmung gewissermaßen getrennt von der textuellen Wahrnehmung betrachtet, in dem sie auf die beiden unterschiedlichen Codes des Rezeptionsprozesses hinweist, versucht die Rezeptionsästhetik die Wahrnehmung eines Textes als einen einheitlichen Prozess zu fassen, und fokussiert dabei besonders auf die Umstände der Textrezeption. Wie in diesem Beitrag gezeigt wird, kann man aber diese beiden, anscheinend unterschiedlichen Herangehensweisen ergänzend verwenden und die Erkenntnisse beider Ansätze im Literatur- und Leseunterricht effektiv nutzen.

Wieoben erwähnt, können bei derVerwendung von Visualisierungen im Literaturund Leseunterricht auch die theoretischen Ansätze der literaturwissenschaftlichen Rezeptionsästhetik (etwa in den theoretischen Schriften von Wolfgang Iser 1972, 1976) zur Geltung gebracht werden. Mit der Rezeptionstheorie akzentuieren Herbert 
Jauß und Wolfgang Iser den produktiven Wahrnehmungsprozess des Lesers und dessen Bedingungen (Iser 1972, 1976; Jauß 1991, 1970). Die Grundannahme, die hinter dem Ansatz steht, ist, dass ein Text verschiedene Interpretationsmöglichkeiten bietet, die sich bei unterschiedlichen Lesern auf verschiedene Art und Weise realisieren. Fragen nach dem Werk bleiben in der Rezeptionsästhetik unberücksichtigt, behandelt werden vornehmlich Fragen nach dem Wahrnehmungsprozess, nach dem subjektiv selektiven Leseverfahren, nach Verständnishorizonten, die das Kunstwerk stillschweigend oder explizit voraussetzt. Bei literarischen Werken versucht der Literaturwissenschaftler, die gesamte Rezeptionsgeschichte, also die prinzipiell mit dem Text gegebenen Lektüremöglichkeiten, zu identifizieren und bestimmte Interpretationen im Spektrum der möglichen Interpretationen weiter zu begründen bzw. in Frage zu stellen. Beim Leseunterricht werden die Lektüremöglichkeiten durch die Interpretationskompetenzen der Studierenden (die Text-Visualisierungen eines jeden Studierenden) beschränkt, was, wie in diesem Text gezeigt wird, weitere Vorteile für das tiefere Textverständnis und eine didaktisierte Textanalyse bringen kann.

Im deutschsprachigen Raum hat die allgemeinen Grundvoraussetzungen für die Verwendung von Visualisierungen im Leseunterricht Erich Hartmann eingeführt und erläutert (Hartmann 2006). Hartmann nimmt einen in verschiedenen Schritten ablaufenden Vorgang an, bei dem das Visualisieren schriftunabhängig an einzelnen Begriffen, Objekten und Szenen, schließlich an Handlungen und Vorgängen geübt wird. Die systematische Einteilung der Vorgänge bei der Verwendung von Visualisierungen scheint auch für diesen Beitrag sinnvoll, denn die weiter unten beschriebene Vorgehensweise dient zum Kennenlernen des Textes, also zur Unterstützung in der ersten Phase der Textanalyse, und fällt damit unter die Kategorie der Visualisierung einzelner Begriffe, Objekte und Szenen, wodurch das Globalverstehen gefördert, aber auch, wie im vorliegenden Beitrag behauptet, eventuellen Missverständnissen und Missinterpretationen vorgebeugt wird.

Der im vorliegenden Beitrag skizzierte Entwurf schließt sich der DCT an und grenzt sich gegenüber anderen Methoden, etwa dem von Nancy Bell entwickelten stark instruktionalistischen Lernprogramm mit ausgearbeiteten externen Visualisierungen und Begleitmaterialien ab. Ganz im Gegenteil stellt beim vorliegenden Entwurf die Spontaneität der eigenen internen Visualisierungen der Lernenden die Grundvoraussetzung für einen erfolgreichen Unterricht dar. Dabei ist eines der Ziele, in der ersten Phase die spontanen Visualisierungen der fortgeschrittenen und erfahrenen Leser $\mathrm{zu}$ erfragen und miteinander $\mathrm{zu}$ konfrontieren. Leseanfänger und -anfängerinnen sowie sehr junge Leser eignen sich für den hier entworfenen Ansatz dagegen weniger: Wie sich auch aus den Studien von Mark Sadoski und Allan Paivio ergibt, sollte diese Gruppe immer erst nach dem für sie mühsamen Erlesen der Worte und Sätze zu Visualisierungen angeregt werden (Sadoski und Paivio 2001: 180). Bei älteren Schülern und Studierenden wird 
dagegen vorausgesetzt, dass sie in der Lage sind, ihre internen Visualisierungen in Worte zu fassen. Aufgrund dieser Fähigkeit können sie aktiv am gemeinsamen Lesen von literarischen Texten teilnehmen. Dabei bleibt aber die Rolle der Lehrperson bei der gezielten Förderung des Leseverstehens erhalten. Damit hängt auch das erforderliche Sprachniveau der Lesenden zusammen, das zwar bei der allgemeinen Verwendung von Visualisierungen im Unterricht keine Rolle spielt, wohl aber beim Lesen und Analysieren von literarischen Kurztexten berücksichtigt werden muss. Empfohlen wird beim hier beschriebenen Ansatz mindestens die Niveaustufe B1 nach dem GER; der Ansatz kann allerdings sowohl im muttersprachlichen als auch im fremdsprachlichen (Lese-) Unterricht verwendet werden.

Es bietet sich an, von der gegenseitigen Konfrontation der individuellen Lektüremöglichkeiten im Lese- und Literaturunterricht zu profitieren, diese weiterzuverarbeiten und sich mit ihnen auseinanderzusetzen, wobei vorausgesetzt wird, dass die Konfrontation mit jeweils alternativen Lektüremöglichkeiten zu einem besseren Textverständnis führt. Außerdem ist zu erwarten, dass bei einem solchen Vorgehen die Literaturkenntnisse der Lesenden spontan erweitert werden. Die positiven Auswirkungen des Visualisierungsprozesses auf das menschliche Gehirn und Gedächtnis werden im weiteren Fortgang des Textes dagegen weniger untersucht, im Fokus steht eher der komplexe Prozess der schematischen und selektiven Externalisierung von mentalen Bildern als kognitive, Übersetzung' von mentalen Bildern in Worte und vor allem in sichtbare Bilder.

\section{Externalisierung von inneren Visualisierungen}

Unstrittig ist, dass beim Lesen mentale Bilder, interne Visualisierungen, entstehen, die die mentalen Fähigkeiten des jeweiligen Menschen stärken und entwickeln. Schon vor mehr als vierzig Jahren kennzeichnet Wolfgang Iser (Iser 1976) den Text als „Partitur“, die im „wandernden Blickpunkt des Lesers“ erschlossen und gestaltet wird. Damit gewinnt die Vorstellungskraft des Lesers beim Interpretationsprozess an Bedeutung. Der Leser ruft im „Akt des Lesens“ Wissen auf, er verstrickt sich mit dem Text, „indem er Erfahrungen erinnert und Vorstellungen bildet“ (Iser 1976: 222). Das Bild, das beim Lesen im Bewusstsein entsteht, ist „die zentrale Kategorie der Vorstellung“. Iser grenzt dieses innere Bild scharf gegen die Objektwahrnehmung eines externen Bildes, beispielsweise gegen die Bilder eines Films, ab, weil diese einen höheren Bestimmtheitsgrad haben und „[...] sich ,als Ganzes' im Blickfeld des Rezipienten befinden, nicht erst im Prozess der Dekodierung des betreffenden Kunstwerkes erzeugt werden“ (Iser 1976: 177). Wie in der Einleitung zum Artikel bereits festgestellt, sind ähnliche Positionen wie die von Iser auch in den neuropsychologischen Studien, etwa den schon erwähnten Arbeiten von Mark Sadoski und Allan Paivio (2012), die sich auf die Entstehung von mentalen Bildern beim Lesen konzentrieren, zu finden. 
Wie bereits eingangs erwähnt, werden Bilder in der deutschen und tschechischen Germanistik grundsätzlich als weniger wertvoll und weniger wirksam betrachtet. So spricht etwa Mechthild Dehn von einem „logozentrischen Stolz“ der Germanistik (Dehn 2007). Sie stellt ebenfalls fest, dass die „Bilderwelten im Kopf“ der Lesenden (Theunert 2006) im Deutschunterricht selten beachtet werden. Der Vorteil der logozentrischen Herangehensweise ist unter anderem die Förderung der Vorstellungskraft und der Fähigkeit abstrakten Denkens. Dieser Vorteil kehrt sich allerdings in der Praxis oft in sein Gegenteil um, wenn nämlich nur Studierende mit starkem Abstraktionsvermögen in der Lage sind, dem roten Faden des Unterrichtsgeschehens zu folgen. Im Extremfall kann es dazu kommen, dass sich die Analyse in abstrakten Modellen verliert und den Text nicht (mehr) berücksichtigt. Die Methode, die im gegenwärtigen Artikel vorgestellt wird, soll dagegen zur Einbeziehung aller Studierenden in den Analyseprozess des jeweiligen literarischen Textes beitragen. Durch die Externalisierung der mentalen Bilder im Zuge einer schrittweisen bildlichen Darstellung werden einerseits wichtige Facetten der jeweiligen Interpretationen der einzelnen Studierenden hervorgehoben, andererseits werden diese durch mögliche Konfrontation und Abstimmung mit anderen in der Gruppe genannten Interpretationsmöglichkeiten vereinigt. Möglicherweise können durch die Externalisierung auch Widersprüche und Ungereimtheiten im Text zu Tage treten, was zu einem tieferen Textverständnis bei den Lernenden führen kann. Der Ansatz wirkt sich sicherlich bei verschiedenen Lernenden unterschiedlich aus, aber es ist zu erwarten, dass die Verwendung von externalisierten Visualisierungen, die Transformation der mentalen Bilder in Worte und schließlich die Externalisierung von internen Bildern eine effektive Ergänzung zur Leseanalyse darstellen ja, sogar manchen Lernenden helfen könnte, den jeweiligen Text überhaupt erst zu verstehen und, wie dies wünschenswert wäre, in Zukunft eine eigene Lesestrategie zu entwickeln.

Es gibt zahlreiche Methoden, eine Externalisierung von mentalen Bildern zu erreichen; in diesem Artikel wird eine vom Kursleiter geleitete Unterrichtsstrategie beschrieben, die die individuellen Abweichungen (idealerweise) in einer einzigen bildlichen Darstellung vereinigt, die im Verlauf des Analysenprozesses allmählich entsteht.

\section{Interpretationsanalysen der Kurzerzählung Das Bette/weib von Locarno in der Literaturwissenschaft - das Rache-Strafe-Paradigma}

Bei einer erfolgreichen Externalisierung von mentalen Bildern im Literaturunterricht spielt eine geeignete Textauswahl die wichtigste Rolle. Bei den ersten Versuchen, innere Visualisierungen im Leseunterricht zu externalisieren, eignen sich Texte, die konkrete Situationen beschreiben, die in einer einfachen Skizze veranschaulicht werden können, also beispielsweise Krimi-Erzählungen, Spukgeschichten, oft 
auch Legenden. Im Allgemeinen handelt es sich also um Kurztexte/Erzählungen, die gattungsabhängig nur einer einzigen linearen Handlung folgen, mit einfacher Figurenkonstellation arbeiten und im Idealfall an einem einzigen Ort spielen, so dass sich auch die mentalen Bilder der Lesenden nur auf einen Ort konzentrieren. Auf diese Weise kann angenommen werden, dass auch die Konfrontation der externalisierten mentalen Bilder untereinander zu einer konstruktiven Diskussion führt und bei der literarischen Textanalyse tatsächlich hilft. Später können auch kompliziertere oder abstraktere Texte extern visualisiert werden. Oft ist es auch günstig, den Visualisierungsprozess an einem konkreten Interpretationsziel auszurichten.

Für den vorliegenden Entwurf einer Visualisierung im Leseunterricht wurde aus mehreren Gründen die Erzählung Das Bettelweib von Locarno von Heinrich von Kleist gewählt. Zum einen gehört sie zu den beliebtesten, in Leselehrbüchern häufig analysierten Erzählungen. Deshalb liegen zu dieser Erzählung sowohl eine Menge von allgemein zugänglichen Interpretationsentwürfen für den Literaturunterricht als auch viele wissenschaftliche Einzelstudien vor. Auf der anderen Seite wird in der Literaturwissenschaft seit vielen Jahren eine Debatte zu einer textgerechten Interpretation dieser Erzählung geführt. Oft wird der Inhalt der Erzählung vereinfacht nach dem Muster von Untat und Strafe, Verbrechen und Rache interpretiert. Im Prisma dieser Interpretationsweise wäre die Erzählung folgendermaßen nachzuerzählen: Eine Marquise lädt eine Bettlerin in ihr Schloss ein und bietet ihr ein Zimmer an. Der Marchese, von der Jagd nach Hause gekommen, befiehlt „der Frau unwillig, aus dem Winkel, in welchem sie lag, aufzustehn und sich hinter den Ofen zu verfügen“ (Kleist: 1810). Die Frau, als sie quer durch das Zimmer zum Ofen geht, „sinkt hinter dem Ofen aber unter Stöhnen und Ächzen nieder und stirbt“ (Kleist, 1810). Später erwacht das Gespenst der Bettlerin zu neuem Leben und rächt sich, indem es den Verkauf des Schlosses durch die Einschüchterung potentieller Käufer verhindert. Schließlich wird der Marchese verrückt und begeht Selbstmord, indem er das ganze Schloss in Brand setzt und selbst in den Flammen umkommt. Dies sei die Strafe, so die traditionelle Interpretation, die der Marchese selbst an sich vollstreckt.

Schon beim gründlicheren Lesen der Erzählung wird klar, dass diese Interpretation lückenhaft ist und viele Details unberücksichtigt lässt. Darauf hat u. a. Johannes F. Lehmann (2006) aufmerksam gemacht. In seinem Text wies er überzeugend nach, dass die Verbrechen-Rache-Interpretationen viele Motive der Erzählung gewaltsam an dieses Schema anpassen oder gar ganz übersehen. Lehmann stützt seine Argumentation besonders auf die vielen leeren Stellen, die bei der Lektüre auf der Grundlage eines Verbrechen-Rache-Musters notwendigerweise entstehen. So hinterfragt er konsequent die mitleidige Tat der Marquise, die in der Exposition der Erzählung steht. Er weist auf den Widerspruch hin, dass die Marquise eine alte und kranke Bettlerin in ein repräsentatives Zimmer im Schloss führt und sie dort auf Stroh bettet. Zudem lässt sie die gehbehinderte Frau die Treppe 
zum Repräsentationszimmer hochsteigen. Lehmann macht ebenfalls darauf aufmerksam, dass das besagte Zimmer zugleich jenes Fremdenzimmer ist, in dem später ein potentieller Käufer vom Wert des Schlosses überzeugt werden soll und das gleichzeitig als „leerstehend“ und „schön und prächtig eingerichtet“ beschrieben wird (Lehmann 2006: 59). Die Inkonsequenz der Handlung der Marquise überrascht den Leser tatsächlich an mehreren Stellen im Text. Aufgrund der Widersprüche im Text folgert Lehmann, dass die "gute Tat" der Marquise aus guten Gründen zu bezweifeln sei, denn, so Lehmann, der Marquise gehe es mit ihrer Mitleidshandlung offensichtlich gar nicht vorrangig um deren Wert für die Empfängerin, sondern um „die Repräsentationsseite des Mitleids, um die Theatralik der Geste, wobei ihr die Kategorien des Verhaltens gegenüber Bettlern durcheinander geraten" (Lehmann 2006: 61). Dies ist auch verkürzt die Schlussfolgerung, die Lehmann zieht: Die Erzählung behandelt eine Geste ohne Mitleid, eine rein „repräsentative“ und somit leere Geste.

Der Ansatz von Lehmann ist nicht nur für die Analyse der Erzählung und für die Kleist-Forschung generell wichtig, sondern gibt Anstoß zu Fragen nach möglichen neuen Lesestrategien, durch die mögliche Leerstellen bei der Analyse besser entdeckt, im Unterricht veranschaulicht und erklärt werden könnten.

\section{Externalisierung der internen Visualisierungen in Das Bettelweib von Locarno}

Sowohl bei Lehmann und anderen Literaturwissenschaftlern als auch für den in diesem Beitrag vorgelegten Entwurf spielt das Stutzig-Werden durch die scheinbare Widersprüchlichkeit des Textes eine entscheidende Rolle und ist, wie auch sonst häufig, die Grundvoraussetzung für kritisches Lesen und letztendlich auch für eine erfolgreiche Analyse. Dazu soll die Externalisierung von internen Visualisierungen der Lesenden und ihre anschließende gegenseitige Abgleichung und Besprechung beitragen. Nachfolgend wird der konkrete Visualisierungsentwurf beschrieben, der zu der Erzählung Das Bettelweib von Locarno in einem Literaturkurs an der Pädagogischen Fakultät der Masaryk-Universität Brno im Wintersemester 2018 mit einer Gruppe von 10 Studierenden durchgeführt wurde.

Wie erwartet werden könnte, hängt der Erfolg der Externalisierung von den internen mentalen Bildern der Lernenden ab, die bereits über Kenntnis des Originaltextes verfügen. Als die Lernenden nach dem ersten Lesen aufgefordert wurden, das Schloss, das in der Erzählung vorkommt, zu zeichnen, waren das Ergebnis grundsätzlich sehr ähnliche Bilder. Zwei Lernende aus einer Gruppe von zehn zeichneten eine Ruine mit weißen Knochen und machten auf den Widerspruch aufmerksam, dass sich „weiße glänzende Knochen in einer alten Schlossruine“" befinden (vergleiche Abb. 1 und Abb. 3). Schon diese Erkenntnis könnte Stoff zu einer Diskussion bieten. Sie gibt unter anderem zu der Frage Anlass, warum 
sich einige Lernende auf die erzählte Geschichte konzentrierten und ein prächtiges Schloss zeichneten, während andere das Resultat der Erzählung (die Ruine mit Knochen) am wichtigsten fanden.

Beim zweiten Lesen wurden die Lernenden aufgefordert, sich durchgehend auf ihre eigenen "mentalen Bilder“ zu konzentrieren, die sie möglicherweise visualisieren könnten. Anschließend wurde versucht, das Schloss nach der Erzählung gemeinsam zu visualisieren und die einzelnen Bildteile mit Textverweisen zu versehen, wobei der Kursleiter als Hinweis erwähnte, die Lernenden könnten sich auch auf die „scheinbaren“ Widersprüche oder Leerstellen im Text konzentrieren. Der Visualisierungsvorgang wurde zwar vom Kursleiter durch Fragen angeleitet, die Lernenden konnten jedoch selber entscheiden, was wie visualisiert und anschließend durch Textverweise nachgewiesen werden sollte. Dadurch erkannten die Lernenden selbst die Widersprüche im Text, auf die u. a. auch Lehmann aufmerksam macht. Sie zeichneten, wie die alte Frau mit Krücken die Treppe hochsteigen musste (s. Abb. 1). Sie zeichneten zwei verschiedene Visualisierungen des Repräsentationszimmers - einmal leer, nur mit Stroh auf dem Boden, für die Bettlerin, und einmal prächtig ausgestattet für den möglichen Käufer des Schlosses; sie zeichneten das Zimmer nochmals, als darin der Marchese und die Marquise auf separaten Betten übernachteten (s. Abb. 2). Die Visualisierungen mit Textverweisen wurden als Ausgangspunkt für die weitere Diskussion im Plenum verwendet. Alle Visualisierungen konnten effektiv zur Vorbereitung der weiteren Analyse beitragen, nicht nur, weil sie sich aus einer tieferen Auseinandersetzung mit den eigenen mentalen Bildern (Externalisierung der internen Visualisierungen), sondern auch mit dem eigentlichen Text (Textverweise zu den Bildern) ergeben hatten. Anschließend wurde eine Visualisierung an die Tafel gezeichnet, die als Resultat der Diskussion alle für die Gruppe wichtigen Merkmale mit Textverweisen zusammenfasste. Eine mögliche Weiterarbeit mit den externalisierten Visualisierungen wäre, den Lernenden eine Hausarbeit aufzugeben, bei der sie den Text von Lehmann zu Hause lesen und eigene Visualisierungen mit den Feststellungen im wissenschaftlichen Text abgleichen könnten.

\section{Externalisierung der internen Visualisierungen - mögliche didaktische Entwürfe}

Aus dem oben beschriebenen Unterrichtsgeschehen und aus nachträglichen Überlegungen ergaben sich zwei mögliche didaktische Entwürfe.

Der erste mögliche Visualisierungsprozess wird vom Kursleiter durchgeführt, d. h. sie/er selbst zeichnet an die Tafel eine Visualisierung, wobei ihm/ihr die Lernenden Hinweise geben, was sie/er zeichnen soll. Am Anfang stehen die Fragen des Kursleiters: „Wie sah das Schloss aus? Zeichnen wir zusammen das Schloss des Marchese.“ „Wo befindet sich das Zimmer?“ Beim Erstellen des Bildes ist es wichtig, 
die einzelnen Hinweise immer textgestützt zu erfragen und somit zu einer plausiblen Visualisierung des Schlosses zu gelangen, damit die gemeinsame „Detektivarbeit“ anschließend erfolgreich fortgesetzt werden kann. Gut ist ebenfalls, zu einzelnen Teilvisualisierungen die Zeilennummern aus dem Text anzuführen, damit die Lernenden jederzeit wissen, auf welche Textstelle sich der jeweilige Bildteil stützt. Bei der Visualisierung ist es günstig, von konkreten Tatsachen, die einfacher erfragt werden können, auszugehen (Wo befindet sich das Zimmer? Wie sieht es aus? Abb. 1) und dann langsam zu schwierigeren Fragen (Welche Textstellen spiegeln wahrscheinlich die eigentliche Beziehung zwischen dem Marchese und der Marquise wider? - Abb. 2) zu kommen. Bei einer erfolgreichen Externalisierung kommt es zu Diskrepanzen und Konfrontationen zwischen verschiedenen ,mentalen Bildern der Lernenden, die bei einer Diskussion (in der Fremdsprache im DaF-Unterricht) erwünscht ist. Wichtig ist, dass mit der Visualisierung auf konkrete, textgestützte Tatsachen hingewiesen wird, die die Argumentation der Lernenden unterstützen und teilweise auch regeln, so dass der Kursleiter bestenfalls nur sehr wenig oder gar nicht in die Diskussion eingreifen muss. Sie/er behält dabei aber das letzte Wort, weil sie/er entscheidet, was am Ende der Diskussion gezeichnet und mit welchen Textstellen dies begründet wird.

Der zweite mögliche Visualisierungsprozess stützt sich auf die selbstständige Arbeit der Lernenden. Dabei gibt es eine ganze Reihe von Möglichkeiten, die, dem Modell von Sadoski entsprechend, auf der Achse zwischen dem skills approach und dem holistic approach zu positionieren sind. Einerseits können die Lernenden einfach ihre beim Lesen entstandenen mentalen Bilder zu Papier bringen und dann miteinander vergleichen. Sie können aber auch Fragen nach den mentalen Bildern vorbereiten, die sie sich gegenseitig stellen und dann versuchen, sie visuell durch Bilder zu beantworten. Die Fragen kann allerdings auch der Kursleiter im Einklang mit dem Ziel seiner Unterrichtsstunde stellen. Grundsätzliche Bedingung für alle Vorgehensweisen (sei es ganzheitlich oder instruktionalisiert) ist aber der textgestützte Vergleich der dabei entstehenden Bilder oder ihrer Entwürfe, die, wie hier angenommen, die Argumentations- und Interpretationsfähigkeit der Lernenden wesentlich fördert. Dabei ist wichtig, dass die Fragen, die mit den Bildern beantwortet werden, mit Nachdruck auf im Text auffindbare konkrete Tatsachen gestellt werden, wobei Visualisierungen von konkreten Tatbeständen einen guten und für die Lernenden verständlichen Ausgangspunkt für etwas kompliziertere, abstraktere Interpretationen des Textes bilden.

\section{Fazit}

Dieser Beitrag befasst sich mit der Verwendung von Visualisierungen im Literaturbzw. Leseunterricht. Im ersten Teil werden theoretische Ansätze aus dem Bereich der Neurologie, Didaktik sowie Literaturwissenschaft vorgestellt, die für die 
Gestaltung eines konkreten Unterrichtsvorschlags relevant sind. Ausgehend von einer Klassifizierung von Visualisierungen nach Erich Hartmann (2006) orientiert sich der in diesem Beitrag beschriebene Entwurf überwiegend an der Visualisierung von einzelnen Begriffen und Objekten und Szenen in der Vorbereitungsphase der eigentlichen Textinterpretation. Trotz der starken Tradition der Germanistik, die sich als schriftorientiertes oder gar "logozentrisches“ Fach profiliert, könnte die Strategie der Verwendung von Visualisierungen - in diesem Artikel konkret die Externalisierung von mentalen Bildern des Lesenden - eine effektive Ergänzung darstellen. Dies v. a. für diejenigen SchülerInnen, die über eine ausgeprägte visuelle Vorstellungskraft verfügen und beim Lesen von Texten eine Menge von mentalen Bildern produzieren, oder auch für diejenigen, die sich bei abstrakten Interpretationen von Texten verloren fühlen. Allgemein konnte beim Testen dieses Entwurfes festgestellt werden, dass durch die angeleitete Externalisierung von mentalen Bildern (mit Textverweisen) das Interesse an einer tieferen Analyse des gelesenen Texten gefördert wird, vor allem weil von den Visualisierungen Impulse zu Diskussionen unter den Lesenden ausgehen können, die zu bis dahin nicht gestellten Fragen nach der Aussage des Textes führen können.

Aus der überwiegend im englischsprachigen Raum ausgearbeiteten DCTTheorie ergab sich die Erkenntnis, dass die Verwendung von Visualisierungen gerade zwischen zwei unterschiedlichen Herangehensweisen zu positionieren ist: zwischen einem Unterrichtsansatz, der auf die Fähigkeit zum Decodieren, zum Verstehen und zur konstruktiven Diskussion abzielt (skills approach), und einem ganzheitlichen Ansatz (holistic approach). Das wurde bei der Ausarbeitung des hier beschriebenen, auf Visualisierungen gestützten Unterrichtsentwurfs berücksichtigt, wobei als Lesematerial die Erzählung Das Bettelweib von Locarno von Heinrich von Kleist diente. Es wurden zwei Alternativen präsentiert: eine durch den Kursleiter moderierte Herangehensweise und eine, die sich stärker auf die selbständige Arbeit der Lernenden stützt. Beim Testen der erstgenannten Alternative ergab sich, dass es effektiver ist, wenn a) die Lernenden den zu visualisierenden Text gut kennen, d. h. ihn wenigstens dreimal gelesen haben, und b) sich die Lernenden während des Lesens auf die eigenen mentalen Bilder konzentrieren, um sie schließlich aufs Papier $\mathrm{zu}$ bringen und über sie zu diskutieren.

\section{Literaturverzeichnis}

Binczek, Natalie/Dembeck, Till/Schäfer, Jörgen (2013): Medien der Literatur, de Gruyter.

Schneider, Lars/Schöch, Christof (2014): Literaturwissenschaft im digitalen Medienwandel, Beiheft 7/2014, PhilN - Philologie im Netz.

Dehn, Mechthild (2007): Unsichtbare Bilder. Überlegungen zum Verhältnis von Text und Bild. In: Didaktik Deutsch 2/2007. S. 25-50. 
Genc, Erhan/Bergmann, Johanna/Singer, Wolf/Kohler Axe (2011): Interhemispheric Connections Shape Subjective Experience of Bistable Motion Current Biology. https://www. mpg.de/4407311/corpus_callosum (10.4.2019).

Hartmann, Erich (2006): In Bildern denken - Texte besser verstehen. München, Ernst Reinhardt.

Hedbávný, Jan (2008): Teorie duálního kódování. M-TIV. http://it.pedf.cuni.cz/ strstud/ edutech/2008_Teorie_dualniho_kodovani_Hedbavny (10.4.2019).

Iser, Wolfgang (1976): Der Akt des Lesens. Theorie ästhetischer Wirkung. München, Fink.

Iser, Wolfgang (1972): Der implizite Leser : Kommunikationsformen des Romans von Bunyan bis Beckett. München, Fink.

Jauß, Hans Robert (1970): Literaturgeschichte als Provokation. Frankfurt am Main, Suhrkamp.

Jauß, Hans Robert (1991): Ästhetische Erfahrung und literarische Hermeneutik. Frankfurt am Main, Suhrkamp.

Jesch, Tatjana/Staiger, Michael (2016): Bilder und Visualisierungen in der Lese- und Literaturdidaktik, In: Gretsch, Petra/Holzäpfel, Lars (Hgg.): Lernen mit Visualisierungen: Erkenntnisse aus der Forschung und deren Implikationen in der Fachdidaktik. Münster, Waxmann. S. 63-82.

Kleists, Heinrich von (1810): Das Bettelweib von Locarno. https://gutenberg.spiegel.de/buch/ das-bettelweib-von-locarno-579/1 (11.6.2019).

Lecke, Bodo (1999): Literatur und Medien in Studium und Deutschunterricht. Berlin [u. a.], Peter Lang.

Lehmann, Johannes F. (2006): Geste ohne Mitleid: Zur Rolle der vergessenen Marquise in Kleists „Das Bettelweib von Locarno“. In: Athenäum 16. S. 57-76.

Lenzner, Alwine (2009): Visuelle Wissenskommunikation: Effekte von Bildern beim Lernen. Kognitive, affektive und motivationale Effekte. Hamburg, Dr. Kovac.

Link, Jürgen (1992): Literatursemiotik. In: Brackert, Helmut/Stückrath, Jörn (Hgg.): Literaturwissenschaft. Ein Grundkurs. Reinbek bei Hamburg, Rowohlt. S. 15-30.

Paivio, Alan (1971): Imagery and Verbal Processes. New York, Holt, Rinehart \& Winston.

Paivio Allan (1986): Mental Representations: A dual coding approach. Oxford, Scholarship.

Sadoski, Mark/Paivio Allan (1994): A dual coding view of imagery and verbal. In: Ruddell, Robert B./Ruddell, Martha R./Singer, Harry (Hgg.): Theoretical models and processes of reading. 4. Aufl. Newark, International Reading Association. S. 582-601.

Sadoski, Mark/Paivio, Allan (2012): Imagery and Text: A Dual Coding Theory of Reading and Writing. New York, Routledge.

Staiger, Michael (2012): Bilder erzählen. Zum Umgang mit visueller Narrativität im Deutschunterricht. In: Oomen-Welke, Ingelore/Staiger, Michael (Hgg.): Bilder in Medien, Kunst, Literatur, Sprache, Didaktik. Festschrift für Adalbert Wichert. Freiburg im Breisgau, Filibach. S. 41-51.

Petr Pytlík

Lehrstuhl für deutsche Sprache und Literatur

Masaryk-Universität Brno/Tschechien

Poříčí $7 / 9$

60300 Brno

E-Mail: pytlik@ped.muni.cz 


\section{ANHANG}

Bilder entstanden während des Kurses „Deutsche Kurzgeschichten“ an der Pädagogischen Fakultät der Masaryk Universität Brünn, Wintersemester 2018. Die Lehrkraft erfragt Informationen von den Studierenden und zeichnet je nach deren Antworten ein Bild.

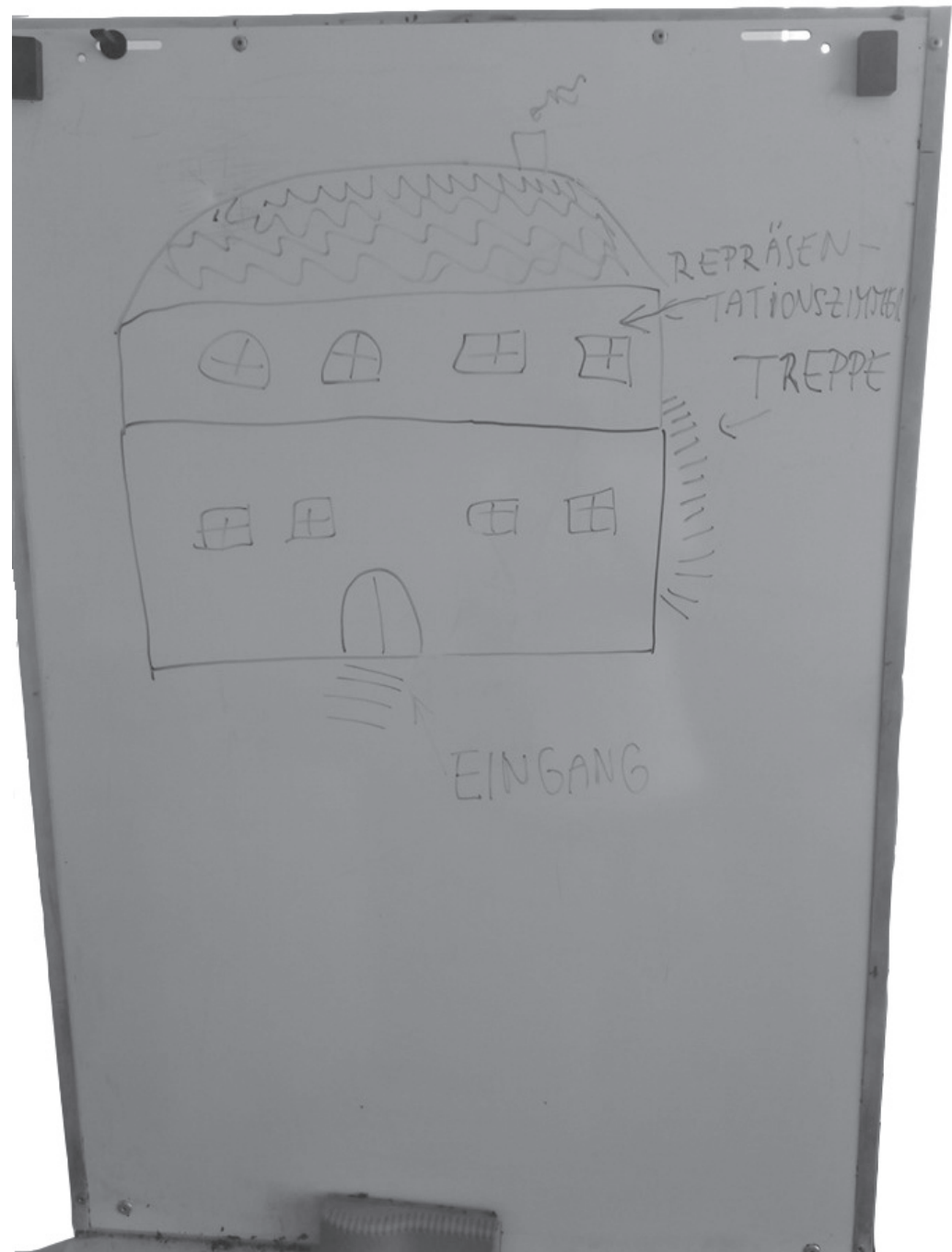

Abb. 1: Anfang der Erzählung - das Schloss mit dem Repräsentationszimmer. 


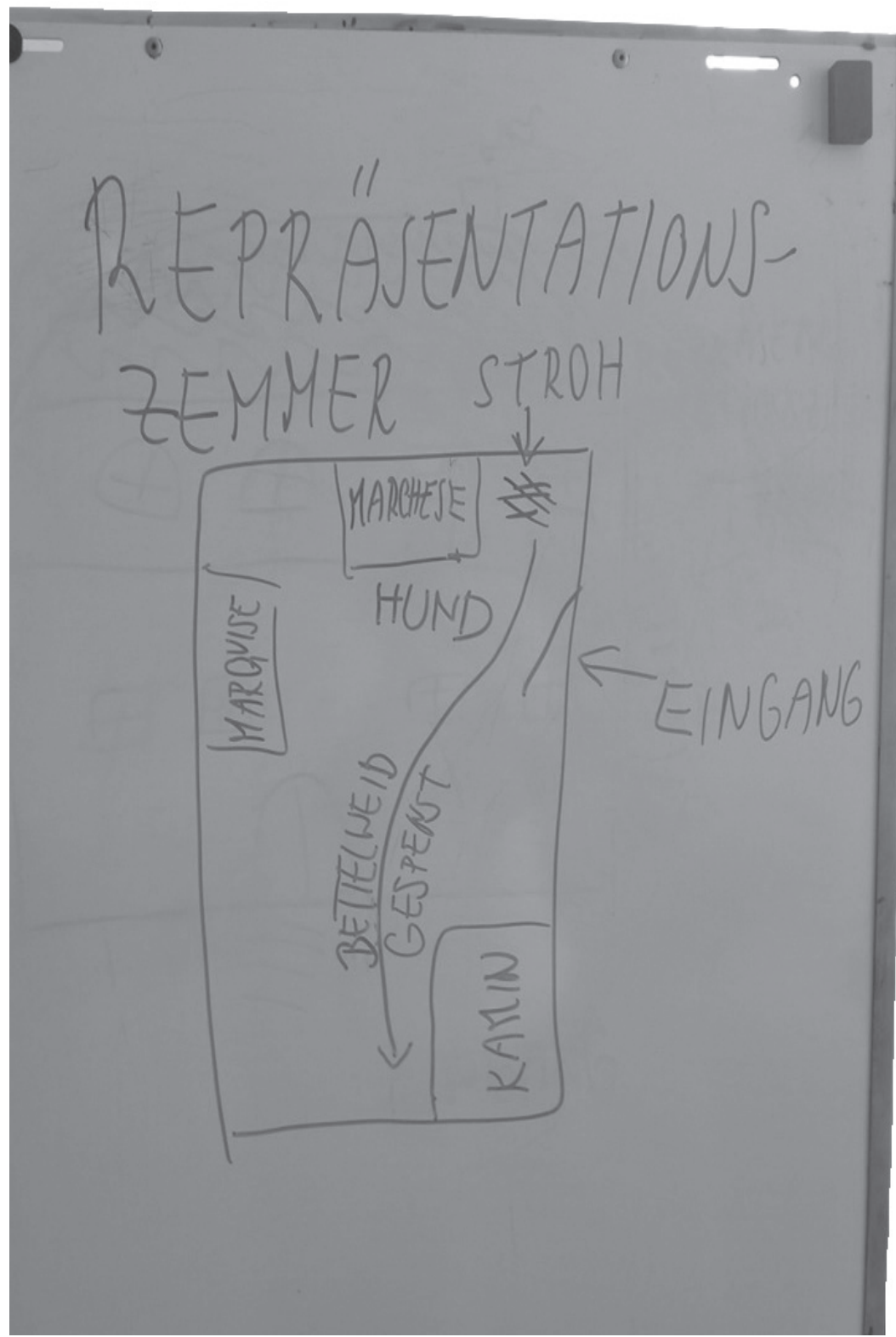

Abb. 2: Nacht - Marchese und Marquise übernachten mit einem Hund im Repräsentationszimmer. 


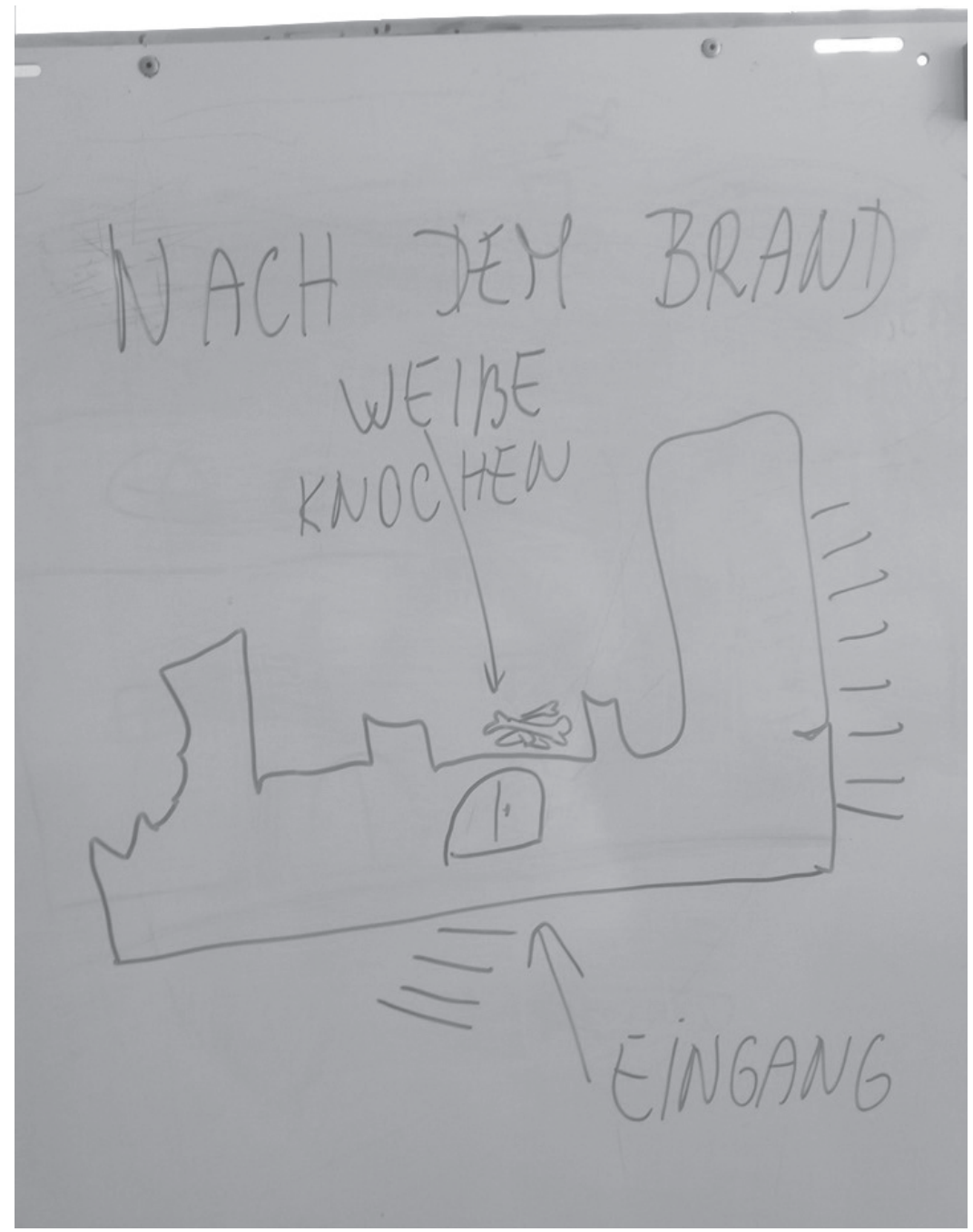

Abb. 3: Schluss der Erzählung - das niedergebrannte Schloss mit den weißen Knochen des Marchese. 